\title{
Normalized Weyl-type «-product on Kähler manifolds
}

\author{
Takuya MASUDA* \\ Department of Physics, Tokyo Metropolitan University, \\ Hachioji, Tokyo, 192-0397, Japan
}

November 13, 2018

\begin{abstract}
We define a normalized Weyl-type $\star$-product on general Kähler manifolds. Expanding this product perturbatively we show that the cumbersome term, which appears in a Berezin-type product, does not appear at least in the first order of $\hbar$. This means a normalization factor, which is introduced by Reshetikhin and Takhtajan for a Berezin-type product, is unnecessary for our Weyl-type product at that order.
\end{abstract}

\section{Introduction}

A new kind of mathematics is thought necessary for a non-perturbative description of the string theory just as Riemannian geometry is indespensable for the description of the theory of general relativity. Non-commutative geometry is tone of the strong candidate for it.

We would like to construct a non-commutative manifold with a Kähler metric from this perspective. We take deformation quantization approach, which introduces a non-commutative product into the ring of functions on a commutative manifold. Kontsevich showed how to construct a non-commutative product on Poisson manifolds, which includes Kähler manifolds [1] [2], but the effect of a metric is implicit in their construction. Dependence of a non-commutativity on a metric is clear in our construction. We give an outline of our construction in the rest part of this section.

Among other approaches we focus our attention on two different types of non-commutative products, the Weyl-type and the Berezin-type. The ordinary

*tmasuda@phys.metro-u.ac.jp 


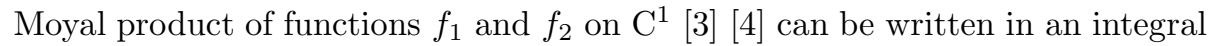
representation, as

$$
\left(f_{1} *_{M} f_{2}\right)(z, \bar{z})=\int_{\mathrm{C}^{1}} f_{1}(w, \bar{v}) f_{2}(v, \bar{w}) \frac{e^{(z \bar{v}+v \bar{z}-z \bar{z}-v \bar{v}) / \hbar}}{e^{(z \bar{w}+w \bar{z}-z \bar{z}-w \bar{w}) / \hbar}} \frac{d v d \bar{v}}{2 \pi i \hbar} \frac{d w d \bar{w}}{2 \pi i \hbar},
$$

which reduces to

$$
f_{1} f_{2}+\frac{\hbar}{2 \sqrt{-1}}\left\{f_{1}, f_{2}\right\}_{P}+O\left(\hbar^{2}\right)
$$

in the small $\hbar$ limit and satisfies associativity. This approach of quantization is applicable only to a flat manifold.

There are papers on Berezin-type star-product on Kähler manifolds 5 [ [6]. The Berezin star-product is defined on certain Kähler manifolds by

$$
\left(f_{1} \bullet f_{2}\right)(z, \bar{z})=\int_{\mathrm{C}^{n}} f_{1}(z, \bar{v}) f_{2}(v, \bar{z}) e^{(\Phi(z, \bar{v})+\Phi(v, \bar{z})-\Phi(z, \bar{z})-\Phi(v, \bar{v})) / \hbar} d \mu_{\hbar}(v, \bar{v})
$$

where $\Phi$ is a Kähler potential of the manifold. This product also satisfies the associativity. The reduction to the Poisson bracket is achieved, however, only in the difference of two terms:

$$
f_{1} \bullet f_{2}-f_{2} \bullet f_{1}=\frac{\hbar}{2 \sqrt{-1}}\left\{f_{1}, f_{2}\right\}_{P}+O\left(\hbar^{2}\right) .
$$

Reshetikhin and Takhtajan showed (1) does not necessarily satisfy $f \bullet 1=$ $1 \bullet f=f$ for the general Kähler potentials and defined a product which satisfies the above equality.

It was shown in [8] that there exists another possible approach

$\left(f_{1} \odot f_{2}\right)(z, \bar{z})=\int_{\mathrm{C}^{n}} f_{1}(w, \bar{v}) f_{2}(v, \bar{w}) \frac{e^{(\Phi(z, \bar{v})+\Phi(v, \bar{z})-\Phi(z, \bar{z})-\Phi(v, \bar{v})) / \hbar}}{e^{(\Phi(z, \bar{w})+\Phi(w, \bar{z})-\Phi(z, \bar{z})-\Phi(w, \bar{w})) / \hbar}} d \mu_{\hbar}(v, \bar{v}) d \mu_{\hbar}(w, \bar{w})$

which interpolates between the Weyl-type and Berezin-type star-products. In the flat space this is the same as the Moyal product. The associativity, however, does not hold by itself, but is fulfilled in the functional integral limit of its multiple products.

Ref. [8] is insufficient in the sense that there is no consideration to the general Kähler manifolds for which $A=\frac{1}{2} \sum_{i, \bar{j}=1}^{n} h^{i \bar{j}} \partial_{i} \bar{\partial}_{j} \log \operatorname{det} H$ is not 0 , where the matrix $H$ is a metric.

We generalize the product defined in [8] so that it is applicable to general Kähler manifolds. Moreover we expand the product perturbatively and show that it really has a property characteristic of a Weyl-type product and that the normalization factor necesarry for a Berezin-type product introduced by Reshetikhin and Takhtajan is unnecessary for a Weyl-type product at least in the first order of $\hbar$. 


\section{Construction}

Reshetikhin and Takhtajan showed the Berezin-type product (11) does not necessarily satisfy $f \bullet 1=1 \bullet f=f$ for the general Kähler potentials and defined, as a product which satisfies the above equality, a normalized star-product

$$
e_{\hbar}^{-1}(z, \bar{z})\left(\left(f_{1} e_{\hbar}\right) \bullet\left(f_{2} e_{\hbar}\right)\right)(z, \bar{z}),
$$

where $e_{\hbar}$ is a normalization factor defined as $f \bullet e_{\hbar}=e_{\hbar} \bullet f=f[$ [7].

As you can see, (2) consists of two kinds of Berezin-type products, an ordinary Berezin-type product

$$
\left(f_{1} \bullet f_{2}\right)(z, \bar{z})=\int_{\mathrm{C}^{n}} f_{1}(z, \bar{v}) f_{2}(v, \bar{z}) e^{(\Phi(z, \bar{v})+\Phi(v, \bar{z})-\Phi(z, \bar{z})-\Phi(v, \bar{v})) / \hbar} d \mu_{\hbar}(v, \bar{v})
$$

and a new kind of Berezin-type product

$\left(f_{1} \circ f_{2}\right)(z, \bar{z}):=\int_{\mathrm{C}^{n}} f_{1}(v, \bar{z}) f_{2}(z, \bar{v}) e^{-(\Phi(z, \bar{v})+\Phi(v, \bar{z})-\Phi(z, \bar{z})-\Phi(v, \bar{v})) / \hbar} d \mu_{\hbar}(v, \bar{v})$.

A normalization factor is necessary for each kind of Berezin-type product, so a normalization factor is also necessary for a Weyl-type product.

We define a normalization factor $\hat{e}_{\hbar}$ for a new kind of Berezin-type product $f_{1} \circ f_{2}$ so that $f \circ \hat{e}_{\hbar}=\hat{e}_{\hbar} \circ f=f$.

$$
e_{\hbar}=1-\hbar A+O\left(\hbar^{2}\right)
$$

whereas

$$
\hat{e}_{\hbar}=1+\hbar A+O\left(\hbar^{2}\right) .
$$

We define a normalized star-product for $f_{1} \circ f_{2}$ just as (3) for $f_{1} \bullet f_{2}$ as

$$
\hat{e}_{\hbar}^{-1}(z, \bar{z})\left(\left(f_{1} \hat{e}_{\hbar}\right) \circ\left(f_{2} \hat{e}_{\hbar}\right)\right)(z, \bar{z}) .
$$

With two kinds of normalization factors $e_{\hbar}$ and $\hat{e}_{\hbar}$ we define a normalized Weyl-type prodet $f_{1} * f_{2}$ as

$$
\begin{aligned}
& \left(f_{1} * f_{2}\right)(z, \bar{z}) \\
:= & e_{\hbar}^{-1}(z, \bar{z})\left(\left(e_{\hbar} f_{1} \hat{e}_{\hbar}\right) \odot\left(e_{\hbar} f_{2} \hat{e}_{\hbar}\right)\right)(z, \bar{z}) \hat{e}_{\hbar}^{-1}(z, \bar{z}) \\
= & \int_{\mathrm{C}^{n}} f_{1}(w, \bar{v}) f_{2}(v, \bar{w}) \frac{e_{\hbar}(w, \bar{v}) e_{\hbar}(v, \bar{w})}{e_{\hbar}(z, \bar{z})} \frac{\hat{e}_{\hbar}(w, \bar{v}) \hat{e}_{\hbar}(v, \bar{w})}{\hat{e}_{\hbar}(z, \bar{z})} \frac{e^{\Phi\left(z^{\prime}, \bar{v}\right)+\Phi(v, \bar{z})-\Phi\left(z^{\prime}, \bar{z}\right)-\Phi(v, \bar{v})}}{e^{\Phi(z, \bar{w})+\Phi\left(w, \bar{z}^{\prime}\right)-\Phi\left(z, \bar{z}^{\prime}\right)-\Phi(w, \bar{w})}} d \mu_{\hbar}(v, \bar{v}) d \mu_{\hbar}(w, \bar{w})
\end{aligned}
$$

This product satisfies

$$
f * 1=1 * f=f
$$

for general Kähler manifolds. It is clear from (ब) generally $f_{1} \odot f_{2} \neq f_{1} * f_{2}$ unless $\hat{e}_{\hbar}=e_{\hbar}^{-1}$. 
As non-normalized star-product $f_{1} \odot f_{2}$, normalized star-product $f_{1} * f_{2}$ does not satisfy the associativity in this form, but the transition to the functional integral version goes as follows.

Multi-products from both sides $\left(f^{(0)} *\left(\cdots *\left(f^{(N-2)} *\left(f^{(N-1)} * f^{(N)}\right)\right) \cdots\right)\right)$ and $\left(\left(\cdots\left(\left(f^{(0)} * f^{(1)}\right) * f^{(2)}\right) * \cdots\right) * f^{(N)}\right)$ are respectively

$$
\begin{aligned}
& \left(f^{(0)} *\left(\cdots *\left(f^{(N-2)} *\left(f^{(N-1)} * f^{(N)}\right)\right) \cdots\right)\right)(z, \bar{z}) \\
= & \int_{\mathrm{C}^{n}}\left(\prod_{j=1}^{N} d \mu_{\hbar}\left(z^{(j-1)}, \bar{v}^{(j)}\right) d \mu_{\hbar}\left(v^{(j)}, \bar{z}^{(j-1)}\right) \frac{e^{\phi\left(v^{(j-1)}, \bar{v}^{(j-1)} ; v^{(j)}, \bar{z}^{(j-1)}\right)}}{e^{\phi\left(v^{(j-1)}, \bar{v}^{(j-1)} ; z^{(j-1)}, \bar{v}^{(j)}\right)}} e_{\hbar}\left(z^{(j-1)}, \bar{z}^{(j-1)}\right) \hat{e}_{\hbar}\left(z^{(j-1)}, \bar{z}^{(j-1)}\right)\right) \\
\times & \frac{e_{\hbar}\left(z^{(N)}, \bar{z}^{(N)}\right)}{e_{\hbar}(z, \bar{z})} \frac{\hat{e}_{\hbar}\left(z^{(N)}, \bar{z}^{(N)}\right)}{\hat{e}_{\hbar}(z, \bar{z})}\left(\prod_{j=0}^{N} f^{(j)}\left(z^{(j)}, \bar{z}^{(j)}\right)\right) \quad\left(v^{(N)}=z^{(N)}, \quad v^{(0)}=z\right), \\
= & \int_{\mathrm{C}^{n}}\left(\prod_{j=1}^{N} d \mu_{\hbar}\left(z^{(j)}, \bar{v}^{(j-1)}\right) d \mu_{\hbar}\left(v^{(j-1)}, \bar{z}^{(j)}\right) \frac{e^{\phi\left(v^{(j)}, \bar{v}^{(j)} ; z^{(j)}, \bar{v}^{(j-1)}\right)}}{\left.e^{\phi\left(v^{(j)}, \bar{v}^{(j)} ; v^{(j-1)}, \bar{z}^{(j)}\right)} e_{\hbar}\left(z^{(j)}, \bar{z}^{(j)}\right) \hat{e}_{\hbar}\left(z^{(j)}, \bar{z}^{(j)}\right)\right)}\right. \\
\times & \frac{e_{\hbar}\left(z^{(0)}, \bar{z}^{(0)}\right)}{e_{\hbar}(z, \bar{z})} \frac{\hat{e}_{\hbar}\left(z^{(0)}, \bar{z}^{(0)}\right)}{\hat{e}_{\hbar}(z, \bar{z})}\left(\prod_{j=0}^{N} f^{(j)}\left(z^{(j)}, \bar{z}^{(j)}\right)\right) \quad\left(v^{(0)}=z^{(0)}, \quad v^{(N)}=z\right) .
\end{aligned}
$$

Therefore they have the same functional integral limit,

$$
\begin{aligned}
& \int \mathcal{D} \mu(z, \bar{v}) \mathcal{D} \mu(v, \bar{z}) \\
\times & \exp \left[\int d \tau\left\{\frac{\partial \bar{v}}{\partial \tau}\left(\frac{\partial \Phi(z, \bar{v})}{\partial \bar{v}}-\frac{\partial \Phi(v, \bar{v})}{\partial \bar{v}}\right)-\frac{\partial v}{\partial \tau}\left(\frac{\partial \Phi(v, \bar{z})}{\partial v}-\frac{\partial \Phi(v, \bar{v})}{\partial v}\right)+\log e_{\hbar}(z, \bar{z})+\log \hat{e}_{\hbar}(z, \bar{z})+\log f(z, \bar{z})\right\}\right] .
\end{aligned}
$$

as in [8], where $f(z, \bar{z})$ is defined as

$$
\int d \tau \log f(z, \bar{z})=\lim _{N \rightarrow \infty} \sum_{j=0}^{N} \frac{1}{N} \log f^{(j)}\left(z^{(j)}, \bar{z}^{(j)}\right) \quad\left(z^{(j)}-z^{(j-1)}=\frac{1}{N}\right) .
$$

¿From the same discussion as in [8], we define a normalized associative Weyltype product as

$$
\begin{aligned}
& f_{1} \star f_{2} \\
= & \int \mathcal{D} \mu(z, \bar{v}) \mathcal{D} \mu(v, \bar{z})
\end{aligned}
$$




$$
\begin{aligned}
\times \quad f_{1}\left(z\left(\tau_{1}\right), \bar{z}\left(\tau_{1}\right)\right) f_{2}\left(z\left(\tau_{1}\right), \bar{z}\left(\tau_{2}\right)\right) \exp \left[\int d \tau\{\right. \\
\left.\left.\quad \frac{\partial \bar{v}}{\partial \tau}\left(\frac{\partial \Phi(z, \bar{v})}{\partial \bar{v}}-\frac{\partial \Phi(v, \bar{v})}{\partial \bar{v}}\right)-\frac{\partial v}{\partial \tau}\left(\frac{\partial \Phi(v, \bar{z})}{\partial v}-\frac{\partial \Phi(v, \bar{v})}{\partial v}\right)+\log e_{\hbar}(z, \bar{z})+\log \hat{e}_{\hbar}(z, \bar{z})\right\}\right],
\end{aligned}
$$

where $\tau_{1}$ and $\tau_{2}$ are fixed points.

\section{Perturbation}

We expand $f_{1} \odot f_{2}$ perturbatively in small $\hbar$. The perturbative expansion of one of the Berezin-type products is given by [7]:

$$
\begin{aligned}
& f_{1} \bullet f_{2} \\
= & \pi^{-n} \operatorname{det} H \int_{\mathrm{C}^{n}} e^{-(H y, y)} \prod_{i=1}^{n} \frac{\left|d y^{i} \wedge d \bar{y}^{i}\right|}{2}\left[f_{1} f_{2}+\epsilon^{2}\left\{y^{i} \bar{y}^{j}\left(\bar{\partial}_{j} f_{1}\right)\left(\partial_{i} f_{2}\right)\right.\right. \\
+ & f_{1} f_{2}\left(-\frac{1}{4} y^{i} y^{j} \bar{y}^{k} \bar{y}^{\ell} \partial_{i} \bar{\partial}_{k} \Phi_{j \bar{\ell}}+y^{i} \bar{y}^{j} \frac{\left(\partial_{i} \bar{\partial}_{j} \operatorname{det} H\right) \operatorname{det} H-\left(\partial_{i} \operatorname{det} H\right)\left(\bar{\partial}_{j} \operatorname{det} H\right)}{(\operatorname{det} H)^{2}}\right) \\
+ & y^{i} \bar{y}^{j}\left(f_{2} \bar{\partial}_{j} f_{1} \frac{\partial_{i} \operatorname{det} H}{\operatorname{det} H}+f_{1} \partial_{i} f_{2} \frac{\bar{\partial}_{j} \operatorname{det} H}{\operatorname{det} H}\right) \\
+ & f_{1} f_{2}\left(\frac{1}{4} y^{i} y^{j} y^{k} \bar{y}^{\ell} \bar{y}^{m} \bar{y}^{n}\left(\partial_{i} \Phi_{j \bar{k}}\right)\left(\bar{\partial}_{\ell} \Phi_{m} \bar{n}\right)+y^{i} \bar{y}^{j} \frac{\left(\partial_{i} \operatorname{det} H\right)\left(\bar{\partial}_{j} \operatorname{det} H\right)}{(\operatorname{det} H)^{2}}\right. \\
- & \left.\left.\frac{1}{2} y^{i} y^{j} \bar{y}^{k} \bar{y}^{\ell}\left\{\frac{\left(\partial_{i} \Phi_{j \bar{k}}\right)\left(\bar{\partial}_{\ell} \operatorname{det} H\right)}{\operatorname{det} H}+\frac{\left(\partial_{i} \operatorname{det} H\right)\left(\bar{\partial}_{k} \Phi_{j \bar{\ell}}\right)}{\operatorname{det} H}\right\}\right)+O\left(\epsilon^{3}\right)\right] \\
= & f_{1} f_{2}+\hbar\left(A f_{1} f_{2}+\sum_{i \bar{j}} h^{i \bar{j}} \bar{\partial}_{j} f_{1} \partial_{i} f_{2}\right)+O\left(\hbar^{2}\right), \quad\left(\hbar=\epsilon^{2}\right)
\end{aligned}
$$

We apply this method of perturbation to the new Berezin-type product, $f_{1} \circ f_{2}$ we have defined above :

$$
\begin{aligned}
& f_{1} \circ f_{2} \\
= & \pi^{-n} \operatorname{det} H \int_{\mathrm{C}^{n}} e^{(H y, y)} \prod_{i=1}^{n} \frac{\left|d y^{i} \wedge d \bar{y}^{i}\right|}{2}\left[f_{1} f_{2}+\epsilon^{2}\left\{y^{i} \bar{y}^{j}\left(\partial_{i} f_{1}\right)\left(\bar{\partial}_{j} f_{2}\right)\right.\right. \\
+ & f_{1} f_{2}\left(\frac{1}{4} y^{i} y^{j} \bar{y}^{k} \bar{y}^{\ell} \partial_{i} \bar{\partial}_{k} \Phi_{j \bar{\ell}}+y^{i} \bar{y}^{j} \frac{\left(\partial_{i} \bar{\partial}_{j} \operatorname{det} H\right) \operatorname{det} H-\left(\partial_{i} \operatorname{det} H\right)\left(\bar{\partial}_{j} \operatorname{det} H\right)}{(\operatorname{det} H)^{2}}\right) \\
+ & y^{i} \bar{y}^{j}\left(f_{1} \bar{\partial}_{j} f_{2} \frac{\partial_{i} \operatorname{det} H}{\operatorname{det} H}+f_{2} \partial_{i} f_{1} \frac{\bar{\partial}_{j} \operatorname{det} H}{\operatorname{det} H}\right)
\end{aligned}
$$




$$
\begin{aligned}
& +f_{1} f_{2}\left(\frac{1}{4} y^{i} y^{j} y^{k} \bar{y}^{\ell} \bar{y}^{m} \bar{y}^{n}\left(\partial_{i} \Phi_{j \bar{k}}\right)\left(\bar{\partial}_{\ell} \Phi_{m \bar{n}}\right)+y^{i} \bar{y}^{j} \frac{\left(\partial_{i} \operatorname{det} H\right)\left(\bar{\partial}_{j} \operatorname{det} H\right)}{(\operatorname{det} H)^{2}}\right. \\
& \left.\left.\left.+\frac{1}{2} y^{i} y^{j} \bar{y}^{k} \bar{y}^{\ell}\left\{\frac{\left(\partial_{i} \Phi_{j \bar{k}}\right)\left(\bar{\partial}_{\ell} \operatorname{det} H\right)}{\operatorname{det} H}+\frac{\left(\partial_{i} \operatorname{det} H\right)\left(\bar{\partial}_{k} \Phi_{j \bar{\ell}}\right)}{\operatorname{det} H}\right\}\right)\right\}+O\left(\epsilon^{3}\right)\right] \\
& =f_{1} f_{2}-\hbar\left(A f_{1} f_{2}+\sum_{i \bar{j}} h^{i \bar{j}} \partial_{i} f_{1} \bar{\partial}_{j} f_{2}\right)+O\left(\hbar^{2}\right) \quad\left(\hbar=\epsilon^{2}\right) .
\end{aligned}
$$

As a result we get

$$
f_{1} \odot f_{2}=f_{1} f_{2}+\hbar \sum_{i, j=1}^{n} h^{i \bar{j}}\left(\bar{\partial}_{j} f_{1} \partial_{i} f_{2}-\partial_{i} f_{1} \bar{\partial}_{j} f_{2}\right)+O\left(\hbar^{2}\right) .
$$

A Poisson bracket appears in the first order of $\hbar$, which means the product $f_{1} \odot f_{2}$ is really a Weyl-type product and which was not shown in [8].

What is surprising is the disapperance of a term $A=\frac{1}{2} h^{i \bar{j}} \partial_{i} \bar{\partial}_{j} \log \operatorname{det} H$ in the first order. Compare this result with the expansion of $f_{1} \bullet f_{2}$ in [7], namely (5), and of $f_{1} \circ f_{2}$. The cumbersome term A, which appears in a Berezin-type product, disappears in a Weyl-type product at least in the order of $\hbar$. This means, therefore, the normalization factor is unnecessary for the Weyl-type product at least in the first order of $\hbar$.

In conclusion we have found that the Weyl-type product defined in [8] can be decomposed into two kinds of Berezin-type products and introduce a normalization factor into a Weyl-type product since a normalization factor is necessary for each kind of Berezin-type product. Note, in general, we must use $f_{1} * f_{2}\left(\neq f_{1} \odot f_{2}\right.$ unless $\left.\hat{e}_{\hbar}=e_{\hbar}^{-1}\right)$.

Moreover we perform a perturbative expansion of non-normalized Weyl-type product. In the first order of $\hbar$ a Poisson bracket appears and $A$ does not appear. Therefore the normalization factor which is necessary for a Berezin-type product is unnecessary for a Weyl-type product at least in the first order of $\hbar$. The normalization factor is unnecessary for Weyl-type product non-perturbatively if $\hat{e}_{\hbar}=e_{\hbar}^{-1}$ is shown.

\section{Acknowledgement}

I would like to thank Dr. Saito for useful discussions and his correction and Dr. Iso for his introduction and explanation of [7] and Mr. Wakatsuki for his cooperation in reading [7].

\section{References}


[1] Maxim Kontsevich, Deformation quantization of Poisson manifolds, q $\operatorname{alg} / 9 \sqrt{09040}$.

[2] Alberto S. Cattaneo and Giovanni Felder, A path integral approach to the Kontsevich quantization formula, Commun.Math.Phys. 212 (2000) 591-611.

[3] J. E. Moyal, Proc. Cambridge. Phil. Soc. 45 (1949) 99.

[4] H. J. Groenewold, Physica, 12 (1946) 405-60.

[5] Michel Cahen, Simone Gutt and John Rawnsley, Trans. AMS, 337 (1993) 73-98

[6] Alexander V. Karabegov, CMP 180 (1996) 745-755

[7] Nicolai Reshetikhin and Leon A. Takhtajan, Deformation Quantization of Kähler Manifolds, math.QA/9907171.

[8] Satoru Saito and Kazunori Wakatsuki, SYMMETRIZATION OF BEREZIN STAR PRODUCT AND PATH INTEGRAL QUANTIZATION, hepth/9912265, Prog. Theor. Phys. 104 No.5 (2000). 